\title{
Pancytopenia-developmental delay syndrome
}

INSERM

\section{Source}

INSERM. (1999). Orphanet: an online rare disease and orphan drug data base.

Pancytopenia-developmental delay syndrome. ORPHA:401764

Pancytopenia-developmental delay syndrome is a rare, genetic, hematologic disorder

characterized by progressive trilineage bone marrow failure (with hypocellularity),

developmental delay with learning disabilities, and microcephaly. Mild facial dysmorphism and hypotonia have also been reported. 